\title{
Modified moments and orthogonal rational functions
}

\author{
J. Van Deun* and A. Bultheel \\ Dept. Computer Science, K.U.Leuven, Celestijnenlaan 200A, B-3001 Heverlee, Belgium
}

\begin{abstract}
In a series of articles $[9,10,11]$ about the numerical computation of orthogonal polynomials on a subset of the real line, Gautschi shows that computing orthogonal polynomials starting from the moments $\mu_{k}=$ $\int x^{k} d \mu(x)$ of the measure is generally an ill-conditioned problem. However, in [10] an alternative approach is presented, based on so-called modified moments, which works better in certain situations. In this paper we generalize these results to the computation of orthogonal rational functions and provide a new modified moment algorithm, based on the connection between modified moments and interpolatory quadrature.
\end{abstract}

\section{Introduction}

The last few years have witnessed a considerable growth in research about orthogonal rational functions, both concerning theoretical properties $[3,7,8,13,14,19,20,21,22,24,27,29]$ as applications in quadrature formulas $[5,6,23]$, continued fractions $[4,26]$, wavelets $[1,2]$ and especially system identification $[16,17,18,30,31,32,33]$. Therefore, the accurate computation of these functions is of major importance. In [25] we presented a general algorithm based on approximating the inner products in the expressions for the recurrence coefficients, similar to what Gautschi calls the discretized Stieltjes procedure for the polynomial case $[9,11,12]$. In this paper we study the possibility of using rational moments of the orthogonality measure to construct orthogonal rational functions.

First we analyze the numerical condition of using ordinary rational moments and show that this is generally an ill-conditioned problem. A possible solution to this problem is the use of rational modified moments. We present two algorithms that use modified moments to compute the recurrence coefficients. The first one is a generalization of the theory that Gautschi developed for the polynomial case [10, 12], but it is limited to the case where all poles are equal to each other. The second algorithm is based on the connection between modified moments and interpolatory quadrature rules and is valid for arbitrary pole sequences.

\section{Preliminaries}

The real line is denoted by $\mathbb{R}$ and the extended real line by $\overline{\mathbb{R}}=\mathbb{R} \cup\{\infty\}$.

By a measure $\mu$ we will mean a positive bounded Borel measure whose support $\operatorname{supp}(\mu) \subset \overline{\mathbb{R}}$ is an infinite set and normalized such that $\mu(\overline{\mathbb{R}})=1$. Since we will only be working with real functions, the inner product in the metric space $L_{2}(\mu)$ can be defined as

$$
\langle f, g\rangle=\int f g d \mu
$$

(there is no need for a complex conjugate bar).

Next we will introduce the spaces of rational functions with real poles. Let a sequence of poles $A=$ $\left\{\alpha_{1}, \alpha_{2}, \ldots\right\} \subset \overline{\mathbb{R}} \backslash\{0\}$ be given such that $A \cap \operatorname{supp}(\mu)=\emptyset$. As a consequence we cannot have that

\footnotetext{
* Corresponding author: e-mail: joris.vandeun@cs.kuleuven.ac.be, Phone: +32 1632 7641, Fax: +32 16327996
} 
$\operatorname{supp}(\mu)=\overline{\mathbb{R}}$. Define factors

$$
Z_{n}(z)=\frac{z}{1-z / \alpha_{n}}
$$

and basis functions

$$
b_{0}(z) \equiv 1, \quad b_{n}(z)=b_{n-1}(z) Z_{n}(z), \quad n=1,2, \ldots
$$

Then the space of rational functions with poles in $A$ is defined as

$$
\mathcal{L}_{n}=\operatorname{span}\left\{b_{0}, \ldots, b_{n}\right\} .
$$

Note that we recover the polynomial case if we take all poles equal to infinity. A function $f=\sum_{k=0}^{n} c_{k} b_{k} \in$ $\mathcal{L}_{n} \backslash \mathcal{L}_{n-1}$ will be called monic if the coefficient of $b_{n}$ is equal to 1.

Let $\mathcal{P}_{n}$ denote the space of polynomials of degree at most $n$ and define

$$
\pi_{n}(z)=\prod_{k=1}^{n}\left(1-z / \alpha_{k}\right),
$$

then we may write equivalently

$$
\mathcal{L}_{n}=\left\{p_{n} / \pi_{n}, p_{n} \in \mathcal{P}_{n}\right\} .
$$

Orthogonalizing the canonical basis $\left\{b_{0}, b_{1}, \ldots, b_{n}\right\}$ with respect to $\mu$ we obtain orthogonal rational functions $\left\{\varphi_{0}, \varphi_{1}, \ldots, \varphi_{n}\right\}$.

The orthogonal rational function $\varphi_{n}$ is called regular if its numerator polynomial satisfies $p_{n}\left(\alpha_{n-1}\right) \neq$ 0 . The system $\left\{\varphi_{n}\right\}$ is regular if $\varphi_{n}$ is regular for every $n$. We now mention the most important theorem for the computation of orthogonal rational functions on the real line, which states that they satisfy a three term recurrence relation, analogous to the one for the polynomial case. For orthonormal rational functions, this is theorem 11.1.2 in [3]. Combining this theorem with lemma 11.1.6 in [3], we can state the recurrence relation in terms of monic orthogonal rational functions. We give the theorem without proof.

Theorem 2.1 Put by convention $\alpha_{-1}=\alpha_{0}=\infty$. Then for $n=1,2, \ldots$ the monic orthogonal rational functions $\varphi_{n}$ satisfy the following three term recurrence relation if and only if $\varphi_{n+1}$ and $\varphi_{n}$ are regular,

$$
\varphi_{n+1}(x)=\left(Z_{n+1}(x)-A_{n} \frac{Z_{n+1}(x)}{Z_{n}(x)}\right) \varphi_{n}(x)-B_{n} \frac{Z_{n+1}(x)}{Z_{n-1}(x)} \varphi_{n-1}(x) .
$$

The initial conditions are $\varphi_{-1}(x) \equiv 0, \varphi_{0}(x) \equiv 1$ and the coefficients $B_{n}$ are strictly positive.

Note that the coefficient $B_{0}$ is never used and can be arbitrarily chosen.

If we take all poles outside the convex hull of $\operatorname{supp}(\mu)$, then the system $\left\{\varphi_{n}\right\}$ will be regular and thus the recurrence relation will hold for every $n$. This follows from the fact that in this case the zeros of $\varphi_{n}$ are inside the convex hull of $\operatorname{supp}(\mu)$. Therefore, if $\operatorname{supp}(\mu)$ is connected then $\left\{\varphi_{n}\right\}$ will be regular (because of the assumptions we made on the location of the poles). In the rest of this paper we assume that this is the case (which means that we are in the situation of orthogonality on a halfline or an interval).

We denote the normalized orthogonal rational functions by $\phi_{n}$. It follows from lemma 11.1.6 in [3] that

$$
\left\|\varphi_{n}\right\|=\sqrt{B_{1} B_{2} \ldots B_{n}}
$$

so the relaton between $\phi_{n}$ and $\varphi_{n}$ is given by

$$
\phi_{n}(x)=\frac{\varphi_{n}(x)}{\sqrt{B_{1} B_{2} \ldots B_{n}}} .
$$

Explicit expressions for the recurrence coefficients in terms of inner products are given in the following lemma. 
Lemma 2.2 The coefficients in the recurrence relation (2) are given by

$$
\begin{aligned}
A_{n} & =\frac{\left\langle Z_{n+1} \varphi_{n}, \varphi_{k}\right\rangle-B_{n}\left\langle\frac{Z_{n+1}}{Z_{n-1}} \varphi_{n-1}, \varphi_{k}\right\rangle}{\left\langle\frac{Z_{n+1}}{Z_{n}} \varphi_{n}, \varphi_{k}\right\rangle} \\
B_{n} & =\frac{\left\|\varphi_{n}\right\|^{2}}{\left\|\varphi_{n-1}\right\|^{2}},
\end{aligned}
$$

for $k \leq n$.

Pro of. The relation for $B_{n}$ follows from equation (3). Taking inner products on both sides of (2) with $\varphi_{k}$ for $k \leq n$ and solving for $A_{n}$ yields the first equation.

Later on we will need interpolating rational functions analogous to the Lagrange interpolating polynomials. Assume that $n$ numbers $\left\{x_{n k}\right\}_{k=1}^{n} \subset \mathbb{R}$ are given, all different from each other and such that none of them coincides with any of the poles in $A$. Only the case where $\left\{x_{n k}\right\}_{k=1}^{n}$ are the zeros of $\varphi_{n}(x)$ will be of interest to us. The following lemma is easily checked and we omit the proof.

Lemma 2.3 Let $\left\{x_{n k}\right\}_{k=1}^{n}$ denote the zeros of $\varphi_{n}(x)$ then the unique rational function $L_{n i} \in \mathcal{L}_{n-1}$ which satisfies $L_{n i}\left(x_{n k}\right)=\delta_{i k}$ is given by

$$
L_{n i}(x)=\frac{\varphi_{n}(x)}{\left(x-x_{n i}\right) \varphi_{n}^{\prime}\left(x_{n i}\right)} \frac{1-x / \alpha_{n}}{1-x_{n i} / \alpha_{n}} .
$$

The unique Hermite interpolating rational functions $H_{n i}, \tilde{H}_{n i} \in \mathcal{L}_{n} \cdot \mathcal{L}_{n-1}$ satisfying

$$
\begin{array}{ll}
H_{n i}\left(x_{n k}\right)=\delta_{i k}, & \tilde{H}_{n i}\left(x_{n k}\right)=0, \\
H_{n i}^{\prime}\left(x_{n k}\right)=0, & \tilde{H}_{n i}^{\prime}\left(x_{n k}\right)=\delta_{i k}
\end{array}
$$

are given by

$$
\begin{aligned}
& H_{n i}(x)=L_{n i}^{2}(x)\left[1-2 L_{n i}^{\prime}\left(x_{n i}\right)\left(x-x_{n i}\right) \frac{1-x_{n i} / \alpha_{n}}{1-x / \alpha_{n}}\right], \\
& \tilde{H}_{n i}(x)=L_{n i}^{2}(x)\left(x-x_{n i}\right) \frac{1-x_{n i} / \alpha_{n}}{1-x / \alpha_{n}}
\end{aligned}
$$

The reproducing kernel $k_{n}(x, y)$ for the space $\mathcal{L}_{n}$ is defined by

$$
k_{n}(x, y)=\sum_{k=0}^{n} \phi_{k}(x) \phi_{k}(y)
$$

and the orthonormal rational functions $\phi_{n}$ satisfy a Christoffel-Darboux relation, which we will only need in its confluent form as given in the next theorem from [3, Chap. 11].

Theorem 2.4 The following relation holds between reproducing kernel and orthonormal basis functions of $\mathcal{L}_{n}$ :

$$
f_{n}^{\prime}(x) f_{n-1}(x)-f_{n}(x) f_{n-1}^{\prime}(x)=\frac{k_{n-1}(x, x)}{\sqrt{B_{n}}},
$$

where

$$
f_{k}(x)=\left(1-\frac{x}{\alpha_{k}}\right) \phi_{k}(x)
$$

and the prime means derivative. 
Next we give a theorem about rational gaussian quadrature formulas. It can be found in [3], chapter 11.6 and in more detail in [23].

Theorem 2.5 Let $\left\{x_{n k}\right\}_{k=1}^{n}$ be the zeros of the $n$-th orthogonal rational function $\varphi_{n}$ and let $\left\{\lambda_{n k}\right\}_{k=1}^{n}$ be defined by

$$
\lambda_{n k}=k_{n-1}\left(x_{n k}, x_{n k}\right)^{-1} .
$$

Then the quadrature formula for

$$
I_{\mu}(f)=\int_{-1}^{1} f(x) d \mu(x)
$$

given by

$$
I_{n}(f)=\sum_{k=1}^{n} \lambda_{n k} f\left(x_{n k}\right)
$$

is exact in $\mathcal{L}_{n} \cdot \mathcal{L}_{n-1}=\left\{g h: g \in \mathcal{L}_{n}, h \in \mathcal{L}_{n-1}\right\}$, i.e. $I_{n}(f)=I_{\mu}(f)$ for any $f$ in $\mathcal{L}_{n} \cdot \mathcal{L}_{n-1}$.

In [3, p. 287] it is shown that an equivalent expression for the weights $\lambda_{n k}$ is given by

$$
\lambda_{n k}=\int_{-1}^{1} L_{n k}(x) d \mu(x)
$$

where $L_{n k}(x)$ is the interpolating rational function from lemma 2.3.

Finally we give a simple lemma which will be useful for future reference.

Lemma 2.6 Let $a, b$ and $x$ be given all in $\mathbb{R}$. Then for any $f$ such that (as a function of $t)(a t+b) /(t-$ $x)[f(t)-f(x)] \in \mathcal{L}_{n-1}$ we have

$$
\int \frac{a t+b}{t-x} \varphi_{n}(t) f(t) d \mu(t)=f(x) \int \frac{a t+b}{t-x} \varphi_{n}(t) d \mu(t) .
$$

This holds in particular if $f \in \mathcal{L}_{n-1}$.

Proof. It follows from $\varphi_{n} \perp \mathcal{L}_{n-1}$ that

$$
\int \varphi_{n}(t) \frac{a t+b}{t-x}[f(t)-f(x)] d \mu(t)=0 .
$$

The result is now immediate.

\section{Ordinary moments}

First let us define what is meant by moments in the case of orthogonal rational functions. For the sake of simplicity, we assume that there are no poles at infinity. Let there be $m$ distinct numbers in the sequence of the first $n$ poles

$$
A^{(n)}=\left\{\alpha_{1}, \alpha_{2}, \ldots, \alpha_{n}\right\}
$$

and denote these by $\left\{\tilde{\alpha}_{k}\right\}_{k=1}^{m}$. Assume that the multiplicity of $\tilde{\alpha}_{k}$ in $A^{(n)}$ is $m_{k}$ (this implies $\sum_{k=1}^{m} m_{k}=$ $n)$. Then the sequence

$$
\tilde{A}^{(n)}=\{\underbrace{\tilde{\alpha}_{1}, \ldots, \tilde{\alpha}_{1}}_{m_{1}}, \underbrace{\tilde{\alpha}_{2}, \ldots, \tilde{\alpha}_{2}}_{m_{2}}, \ldots, \underbrace{\tilde{\alpha}_{m}, \ldots, \tilde{\alpha}_{m}}_{m_{m}}\}
$$


will generate the same space $\mathcal{L}_{n}$ as $A^{(n)}$. If we order the $\tilde{\alpha}_{k}$ such that $\alpha_{n}=\tilde{\alpha}_{m}$, then $A^{(n-1)}$ will also generate the same $\mathcal{L}_{n-1}$ as $\tilde{A}^{(n-1)}$. Assume that this has been done. A basis for $\mathcal{L}_{n} \cdot \mathcal{L}_{n-1}$ can then be given by

$$
\left\{\begin{array}{l}
\frac{1}{x-\tilde{\alpha}_{1}}, \frac{1}{\left(x-\tilde{\alpha}_{1}\right)^{2}}, \ldots, \frac{1}{\left(x-\tilde{\alpha}_{1}\right)^{2 m_{1}}}, \\
\frac{1}{x-\tilde{\alpha}_{2}}, \frac{1}{\left(x-\tilde{\alpha}_{2}\right)^{2}}, \ldots, \frac{1}{\left(x-\tilde{\alpha}_{2}\right)^{2 m_{2}}}, \\
\vdots \\
\frac{1}{x-\tilde{\alpha}_{m-1}}, \frac{1}{\left(x-\tilde{\alpha}_{m-1}\right)^{2}}, \ldots, \frac{1}{\left(x-\tilde{\alpha}_{m-1}\right)^{2 m_{m-1}}}, \\
\frac{1}{x-\tilde{\alpha}_{m}}, \frac{1}{\left(x-\tilde{\alpha}_{m}\right)^{2}}, \ldots, \frac{1}{\left(x-\tilde{\alpha}_{m}\right)^{2 m_{m}-1}} .
\end{array}\right.
$$

There are obviously $2 n$ functions in this basis, which we denote by $\left\{p_{k}\right\}_{k=0}^{2 n-1}$. So we have

$$
\begin{aligned}
& p_{0}(x) \equiv 1, \\
& p_{i}(x)=\left(x-\tilde{\alpha}_{k}\right)^{-l}, \quad i=2 \sum_{j=1}^{k-1} m_{j}+l, \quad k=1, \ldots, m .
\end{aligned}
$$

Now the moments $\mu_{k}$ of the orthogonality measure $\mu$ are defined as

$$
\mu_{k}=\int p_{k}(x) d \mu(x), \quad k=0, \ldots, 2 n-1 .
$$

As Gautschi does for the polynomial case, we take the position that the fundamental quantities in the computation of orthogonal rational functions are the recurrence coefficients. Once these are known, the accurate evaluation of the functions is straightforward. Furthermore, these coefficients can be gathered in a tridiagonal matrix to obtain a generalized eigenvalue problem whose solutions are the zeros of $\varphi_{n}$, i.e. the nodes in the quadrature formulas. The weights are defined by the first components of the corresponding eigenvectors. For more information we refer to [6].

Once the moments (5) are known, the recurrence coefficients $A_{k}$ and $B_{k}$ can be computed for $k=$ $0, \ldots, n-1$ by evaluating the inner products from lemma 2.2 by a partial fraction decomposition and integrating term by term. Note that the integrands in the inner products for $A_{n-1}$ and $B_{n-1}$ are in $\mathcal{L}_{n} \cdot \mathcal{L}_{n-1}$.

However, as we will see in this section, computing the coefficients from the moments is usually an ill-conditioned problem. First we define the vectors

$$
\underline{\mu}^{T}=\left[\mu_{0}, \mu_{1}, \ldots, \mu_{2 n-1}\right], \quad \underline{c}^{T}=\left[A_{0}, \ldots, A_{n-1}, B_{0}, \ldots, B_{n-1}\right] .
$$

Now we are interested in the condition of the map $K_{n}: \underline{\mu} \rightarrow \underline{c}$.

It is convenient to think of $K_{n}$ as the composition of two maps,

$$
K_{n}=K_{n}^{(1)} \circ K_{n}^{(2)}
$$

where $K_{n}^{(2)}$ is the map from the moments to the quadrature formula (which is exact in $\mathcal{L}_{n} \cdot \mathcal{L}_{n-1}$ ),

$$
K_{n}^{(2)}: \underline{\mu} \rightarrow \underline{q}, \quad \underline{q}^{T}=\left[x_{n 1}, \ldots, x_{n n}, \lambda_{n 1}, \ldots, \lambda_{n n}\right],
$$


and $K_{n}^{(1)}$ the map from the quadrature formula to the coefficients,

$$
K_{n}^{(1)}: \underline{q} \rightarrow \underline{c} .
$$

The latter map is usually not very sensitive and we do not study it. We focus on the map $K_{n}^{(2)}$, which is the ill-conditioned one. As condition number of a map $M: x \rightarrow y$ from one finite-dimensional space into another we generally adopt the quantity

$$
(\text { cond } M)(x)=\frac{\|x\|\left\|J_{M}(x)\right\|}{\|M x\|}
$$

where $J_{M}$ is the Jacobian matrix of $M$, and $\|\cdot\|$ a suitable vector norm and subordinate matrix norm. The map $K_{n}^{(2)}$ amounts to solving the nonlinear system of equations,

$$
\sum_{i=1}^{n} \lambda_{n i} p_{k}\left(x_{n i}\right)=\mu_{k}, \quad k=0,1,2, \ldots, 2 n-1 .
$$

The Jacobian of the map defined by (6) can easily be computed and equals

$$
J_{K_{n}^{(2)}}^{-1}=T \Lambda,
$$

where

$$
\begin{aligned}
\Lambda & =\operatorname{diag}\left(1, \ldots, 1, \lambda_{n 1}, \ldots, \lambda_{n n}\right), \\
T & =\left[\begin{array}{cccccc}
p_{0}\left(x_{n 1}\right) & \cdots & p_{0}\left(x_{n n}\right) & p_{0}^{\prime}\left(x_{n 1}\right) & \cdots & p_{0}^{\prime}\left(x_{n n}\right) \\
p_{1}\left(x_{n 1}\right) & \cdots & p_{1}\left(x_{n n}\right) & p_{1}^{\prime}\left(x_{n 1}\right) & \cdots & p_{1}^{\prime}\left(x_{n n}\right) \\
\vdots & & \vdots & \vdots & & \vdots \\
p_{2 n-1}\left(x_{n 1}\right) & \cdots & p_{2 n-1}\left(x_{n n}\right) & p_{2 n-1}^{\prime}\left(x_{n 1}\right) & \cdots & p_{2 n-1}^{\prime}\left(x_{n n}\right)
\end{array}\right] .
\end{aligned}
$$

It follows that

$$
\left(\operatorname{cond} K_{n}^{(2)}\right)(\underline{\mu})=\frac{\|\underline{\mu}\|\left\|\Lambda^{-1} T^{-1}\right\|}{\|\underline{q}\|} .
$$

To compute $T^{-1}$ we note that it may be written as

$$
T^{-1}=\left[\begin{array}{l}
T^{(1)} \\
T^{(2)}
\end{array}\right]
$$

with

$$
\begin{array}{ll}
T^{(1)}=\left[t_{i j}^{(1)}\right], & i=1, \ldots, n, \quad j=0, \ldots, 2 n-1, \\
T^{(2)}=\left[t_{i j}^{(2)}\right], \quad i=1, \ldots, n, \quad j=0, \ldots, 2 n-1
\end{array}
$$

and the entries $t_{i j}^{(1)}$ and $t_{i j}^{(2)}$ can be computed from the equation $T T^{-1}=I_{2 n}$ (where $I_{2 n}$ is the identity matrix of order $2 n$ ). This yields the equations

$$
\begin{array}{ll}
\sum_{j=0}^{2 n-1} t_{i j}^{(1)} p_{j}\left(x_{n k}\right)=\delta_{i k}, & \sum_{j=0}^{2 n-1} t_{i j}^{(2)} p_{j}\left(x_{n k}\right)=0, \\
\sum_{j=0}^{2 n-1} t_{i j}^{(1)} p_{j}^{\prime}\left(x_{n k}\right)=0, & \sum_{j=0}^{2 n-1} t_{i j}^{(2)} p_{j}^{\prime}\left(x_{n k}\right)=\delta_{i k},
\end{array}
$$


which is equivalent to saying that

$$
\begin{aligned}
& \sum_{j=0}^{2 n-1} t_{i j}^{(1)} p_{j}(x)=H_{n i}(x), \\
& \sum_{j=0}^{2 n-1} t_{i j}^{(2)} p_{j}(x)=\tilde{H}_{n i}(x),
\end{aligned}
$$

where $H_{n i}$ and $\tilde{H}_{n i}$ are the Hermite interpolating rational functions from lemma 2.3. As in the polynomial case, we can give a lower bound for the condition number, involving orthogonal polynomials, as shown in the following theorem.

Theorem 3.1 The condition number cond $K_{n}^{(2)}$ satisfies

$$
\operatorname{cond} K_{n}^{(2)} \geq c_{n} \frac{\|\underline{\mu}\|}{\|\underline{q}\|} \frac{\lim _{x \rightarrow \infty} \varphi_{n}^{2}(x)}{\min _{1 \leq i \leq n}\left\{\lambda_{n i}\left[\varphi_{n}^{\prime}\left(x_{n i}\right)\right]^{2}\left|x_{n i}-\alpha_{n}\right|\right\}},
$$

where $c_{n}>0$ is a constant only depending on $n$ and the definition of the matrix norm. If we use the 1 -norm (maximum column sum) or the $\infty$-norm (maximum row sum), then $c_{n}=1$. In the case of the interval $[-1,1]$, with poles A staying away from the boundary and the measure $\mu$ satisfying the ErdösTurán condition $\mu^{\prime}>0$ a.e., we get the asymptotic lower bound

$$
\operatorname{cond} K_{n}^{(2)} \gtrsim c_{n} \frac{\|\underline{\mu}\|}{\|\underline{q}\|} \frac{1}{64 \pi} \frac{(1-\rho)^{7}}{n \rho^{2 n-1}},
$$

where $\rho=\sup _{1 \leq k \leq n}\left|\alpha_{k}-\operatorname{sign}\left(\alpha_{k}\right) \sqrt{\alpha_{k}^{2}-1}\right|$. We use the symbol $\gtrsim$ to mean that the inequality only holds for large $n$ (theoretically infinite).

Proof. It follows from the equivalence of norms in a finite-dimensional vector space that for every matrix norm (not necessarily induced by a vector norm)

$$
\|\cdot\|: \mathbb{R}^{2 n \times 2 n} \rightarrow \mathbb{R}^{+}: A \rightarrow\|A\|
$$

there exists a constant $c_{n}>0$ such that for all $A \in \mathbb{R}^{2 n \times 2 n}$ we have

$$
\|A\| \geq c_{n} \max _{1 \leq i, j \leq 2 n}\left|a_{i j}\right|
$$

(where $a_{i j}$ are the elements of $A$ ).

Note that for every $1 \leq i \leq n$ we have $t_{i 0}^{(2)}=\lim _{x \rightarrow \infty} \tilde{H}_{n i}(x)$. The first statement in the theorem now follows from the definition of $\Lambda$, equation (8) and lemma 2.3 .

To obtain the second statement, note that equation (7) can be written in terms of the normalized functions $\phi_{n}$, just replacing $\varphi_{n}(x)$ by $\phi_{n}(x)$ and $\varphi_{n}^{\prime}\left(x_{n i}\right)$ by $\phi_{n}^{\prime}\left(x_{n i}\right)$. We can then use the relation between orthonormal rational functions on the interval $[-1,1]$ and on the complex unit circle, as given in [29]. It follows from theorem 4.1 in [29], theorem 9.8.15 in [3] and the proof of lemma 9.3.1 in [3] that under the conditions on the measure and poles stated above, we have the asymptotic bound

$$
\lim _{x \rightarrow \infty} \phi_{n}^{2}(x) \gtrsim \frac{1}{2 \pi} \frac{1-\beta_{n}^{2}}{\prod_{k=1}^{n} \beta_{k}^{2}}
$$

where $\beta_{k} \in(-1,1)$ and $\alpha_{k}$ are related by the Joukowski transformation,

$$
\alpha_{k}=\frac{1}{2}\left(\beta_{k}+\frac{1}{\beta_{k}}\right) \text {. }
$$


Bounding the denominator (with the derivative of $\phi_{n}$ ) is a bit more complicated, but we can use the confluent Christoffel-Darboux relation from theorem 2.4 and the fact that $k_{n-1}\left(x_{n i}, x_{n i}\right)=\lambda_{n i}^{-1}$ to obtain

$$
\lambda_{n i}\left[\phi_{n}^{\prime}\left(x_{n i}\right)\right]^{2}\left|x_{n i}-\alpha_{n}\right|=\frac{\alpha_{n}^{2} \alpha_{n-1}^{2}}{B_{n} \lambda_{n i}\left|x_{n i}-\alpha_{n-1}\right|^{2} \phi_{n-1}^{2}\left(x_{n i}\right)\left|x_{n i}-\alpha_{n}\right|} .
$$

Because the quadrature formula is exact in $\mathcal{L}_{n} \cdot \mathcal{L}_{n-1}$, we know that

$$
\int_{-1}^{1} \phi_{n-1}^{2}(x) d \mu(x)=\sum_{k=1}^{n} \lambda_{n k} \phi_{n-1}^{2}\left(x_{n k}\right)=1
$$

from which it follows that

$$
\max _{1 \leq i \leq n} \lambda_{n i} \phi_{n-1}^{2}\left(x_{n i}\right)>\frac{1}{n} \text {. }
$$

The quadrature nodes are all contained in $[-1,1]$, so we also have

$$
\left|x_{n i}-\alpha_{k}\right|>\left|\alpha_{k}\right|-1, \quad 1 \leq i \leq n, \quad k=1,2, \ldots
$$

From theorem 7 in [24] it follows that $B_{n} \geq 1 / 4$ for large $n$. Combining all the previous results and using the definition of the Joukowski transformation gives after some computations that

$$
\operatorname{cond} K_{n}^{(2)} \gtrsim c_{n} \frac{\|\underline{\mu}\|}{\|\underline{q}\|} \frac{1}{2 \pi} \frac{1-\beta_{n}^{2}}{\prod_{k=1}^{n-1} \beta_{k}^{2} \beta_{n}} \frac{\left(1-\left|\beta_{n-1}\right|\right)^{4}}{\left(1+\beta_{n-1}^{2}\right)^{2}} \frac{\left(1-\left|\beta_{n}\right|\right)^{2}}{\left(1+\beta_{n}^{2}\right)^{2}} \frac{1}{2 n} .
$$

Using the fact that $\left|\beta_{k}\right| \leq \rho$ and $1 /\left(1+\rho^{2}\right) \geq 1 / 2$ finishes the proof.

Note that the statement we just proved shows that the condition number grows exponentially for large $n$.

Example 3.2 Let us look at the case where we have an exponential weight on $[-1,1]$ and where all poles are equal to each other. We take

$$
d \mu(x)=\exp \left(-\frac{a}{1+x}\right) d x
$$

with $a>0$ a parameter, and

$$
A=\{\alpha, \alpha, \alpha, \ldots\}
$$

where $|\alpha|>1$. We need expressions for the moments

$$
\mu_{k}=\int_{-1}^{1} \frac{\exp \left(-\frac{a}{1+x}\right)}{(x-\alpha)^{k}} d x, \quad k=0,1, \ldots
$$

which can be obtained as follows. Integrating by parts twice gives

$$
\begin{aligned}
\mu_{n}= & \frac{1}{(1-\alpha)^{n}}\left[2 e^{-a / 2}+a \operatorname{Ei}(-a / 2)\right]+n \mu_{n} \\
& +n(1+\alpha) \mu_{n+1}+a n \int_{-1}^{1} \frac{\operatorname{Ei}\left(-\frac{a}{1+x}\right)}{(x-\alpha)^{n+1}} d x \\
= & \frac{2 e^{-a / 2}}{(1-\alpha)^{n}}+n \mu_{n}+n(1+\alpha) \mu_{n+1}-a \int_{-1}^{1} \frac{\exp \left(-\frac{a}{1+x}\right)}{(1+x)(x-\alpha)^{n}} d x
\end{aligned}
$$


Table 1 Condition of the map $K_{n}^{(2)}$

\begin{tabular}{ccc}
\hline$n$ & cond $K_{n}^{(2)}$ & err. growth \\
\hline 2 & $8.1316 e+00$ & $3.0805 e+03$ \\
5 & $8.2203 e+02$ & $2.5416 e+05$ \\
8 & $2.0054 e+04$ & $1.4776 e+08$ \\
11 & $1.8818 e+06$ & $8.9086 e+10$ \\
14 & $3.9708 e+08$ & $8.8312 e+13$ \\
17 & $1.3211 e+11$ & $3.5909 e+15$ \\
20 & $6.7577 e+13$ & $8.0136 e+16$ \\
\hline
\end{tabular}

where $\operatorname{Ei}(z)$ is the exponential integral, defined for real $z<0$ as $\int_{-\infty}^{z} e^{t} / t d t$. Although the second integration by parts is only valid for $n \geq 1$, it is easily checked that the final formula also holds for $n=0$. Putting

$$
\nu_{n}=\int_{-1}^{1} \frac{\exp \left(-\frac{a}{1+x}\right)}{(1+x)(x-\alpha)^{n}} d x
$$

it can be verified that $\nu_{n}=\left(\mu_{n}-\nu_{n-1}\right) /(1+\alpha)$. Substituting back and eliminating $\nu_{n}$ and $\nu_{n-1}$ then yields the recurrence relation

$$
-n(1+\alpha)^{2} \mu_{n+1}=\frac{4 e^{-a / 2}}{(1-\alpha)^{n}}+[2(n-1)(1+\alpha)-a] \mu_{n}+(n-2) \mu_{n-1} .
$$

for $n \geq 1$. The initial values have to be computed explicitly and are

$$
\begin{aligned}
& \mu_{0}=2 e^{-a / 2}+a \operatorname{Ei}(-a / 2) \\
& \mu_{1}=-\operatorname{Ei}(-a / 2)+\operatorname{Ei}\left(-\frac{a}{2} \frac{\alpha-1}{\alpha+1}\right) \exp \left(-\frac{a}{1+\alpha}\right) .
\end{aligned}
$$

With the previous formulas all the moments can be computed. As an example we take $a=4$ and $\alpha=$ -1.01 . Table 1 gives the observed error growth (the largest relative error on the recurrence coefficients divided by the machine precision) and the lower bound for the condition number computed using the first formula of the previous theorem. Note that this bound underestimates the actual error growth.

\section{Modified moments}

As shown in the previous section, computing the recurrence coefficients from the moments is generally an ill-conditioned problem. To overcome this problem in the polynomial case, so-called modified moments were introduced. Here we study the possibility of using modified moments in the construction of orthogonal rational functions. The procedure in this section is analogous to the one for the polynomial case as described in [10]. However, for reasons explained later on, it is required that all poles be equal to each other. In the next section we provide an algorithm based on the relation between modified moments and interpolatory quadrature formulas, which works for arbitrary poles.

It will be convenient to work with the normalized orthogonal rational functions. Because of relation (3) we may rewrite the recurrence relation in terms of the orthonormal functions as

$$
\phi_{n}(x)=\left(E_{n} Z_{n}(x)+F_{n} \frac{Z_{n}(x)}{Z_{n-1}(x)}\right) \phi_{n-1}(x)-\frac{E_{n}}{E_{n-1}} \frac{Z_{n}(x)}{Z_{n-2}(x)} \phi_{n-2}(x)
$$


with the new coefficients $E_{n}$ and $F_{n}$ satisfying

$$
E_{n}=\frac{1}{\sqrt{B_{n}}}, \quad F_{n}=\frac{-A_{n-1}}{\sqrt{B_{n}}} .
$$

This is the recurrence relation used in $[3,23,24,25]$ etc. Now assume that we are given a set of rational functions $\left\{\tilde{\phi}_{n}\right\}$ orthonormal with respect to a measure $\tilde{\mu}$ and with one multiple pole in $\alpha$. The functions we wish to compute are denoted by $\phi_{n}$ and orthonormal with respect to $\mu$. They have the same multiple pole $\alpha$ as the $\tilde{\phi}_{n}$. Furthermore assume that we have at our disposition all the modified moments

$$
\nu_{k}=\int \tilde{\phi}_{k}(x) d \mu(x)
$$

Consider then the Gram matrix $M=\left[m_{i j}\right]$ of order $n+1$ where

$$
m_{i j}=\int \tilde{\phi}_{i}(x) \tilde{\phi}_{j}(x) d \mu(x), \quad i, j=0,1, \ldots, n
$$

and let $M=R^{T} R$ be the Cholesky decomposition of $M$. Putting $S=\left[s_{i j}\right]=R^{-1}$ it follows from [15] that

$$
\phi_{j}(x)=\sum_{i=0}^{j} s_{i j} \tilde{\phi}_{i}(x), \quad j=0,1, \ldots, n .
$$

Substituting this equation into the recurrence relation (9) for the $\phi_{n}$, we get

$$
\begin{aligned}
\frac{x}{1-x / \alpha} \sum_{i=0}^{n-1} s_{i, n-1} \tilde{\phi}_{i}(x)=\frac{1}{E_{n}} \sum_{i=0}^{n} s_{i n} & \tilde{\phi}_{i}(x) \\
& -\frac{F_{n}}{E_{n}} \sum_{i=0}^{n-1} s_{i, n-1} \tilde{\phi}_{i}(x)+\frac{1}{E_{n-1}} \sum_{i=0}^{n-2} s_{i, n-2} \tilde{\phi}_{i}(x)
\end{aligned}
$$

for $n \geq 3$. Because $Z_{-1}(x)=Z_{0}(x)=x$ is different from $Z_{n}(x)$ for $n \geq 1$, we have to modify the above relation when $n<3$, but we leave the details to the reader. The left hand side of (10) can also be written as a linear combination of the $\tilde{\phi}_{i}$, using the recurrence for these functions backwards. We have

$$
\frac{x}{1-x / \alpha} \tilde{\phi}_{i}(x)=\frac{1}{\tilde{E}_{i+1}} \tilde{\phi}_{i+1}(x)-\frac{\tilde{F}_{i+1}}{\tilde{E}_{i+1}} \tilde{\phi}_{i}(x)+\frac{1}{\tilde{E}_{i}} \tilde{\phi}_{i-1}(x) .
$$

Note that this can only be done if all poles are equal to each other. Otherwise we would have to find the coefficients in an expression of the form

$$
\frac{1}{1-x / \alpha_{n}} \tilde{\phi}_{i}(x)=\sum_{k=0}^{n} c_{k}^{(i)} \tilde{\phi}_{k}(x)
$$

which is much more complicated.

Now coefficients of equal $\tilde{\phi}_{i}$ must agree on both sides of (10) because of the linear independence. For $\tilde{\phi}_{n}$ and $\tilde{\phi}_{n-1}$ this yields after some computations

$$
\begin{aligned}
E_{n} & =\frac{s_{n n}}{s_{n-1, n-1}} \tilde{E}_{n}, \\
F_{n} & =\frac{s_{n-1, n}}{s_{n-1, n-1}}+\frac{s_{n n}}{s_{n-1, n-1}} \tilde{F}_{n}-\frac{s_{n-2, n-1} s_{n n}}{s_{n-1, n-1}^{2}} \frac{\tilde{E}_{n}}{\tilde{E}_{n-1}}
\end{aligned}
$$


for $n \geq 3$. These relations can be written in terms of the elements of $R$ using $s_{j j}=1 / r_{j j}$ and $s_{j, j+1}=$ $-r_{j, j+1} /\left(r_{j j} r_{j+1, j+1}\right)$. This means that, once the Gram matrix $M$ is known, we can easily compute the recurrence coefficients for $\phi_{n}$ from the coefficients for $\tilde{\phi}_{n}$. The Gram matrix can be constructed from the modified moments by applying the recursion twice as follows.

$$
\begin{aligned}
m_{i j}= & \left\langle\tilde{\phi}_{i}, \tilde{\phi}_{j}\right\rangle \\
= & \tilde{E}_{i}\left\langle\frac{x}{1-x / \alpha} \tilde{\phi}_{i-1}, \tilde{\phi}_{j}\right\rangle+\tilde{F}_{i}\left\langle\tilde{\phi}_{i-1}, \tilde{\phi}_{j}\right\rangle-\frac{\tilde{E}_{i}}{\tilde{E}_{i-1}}\left\langle\tilde{\phi}_{i-2}, \tilde{\phi}_{j}\right\rangle \\
= & \tilde{E}_{i}\left\langle\tilde{\phi}_{i-1}, \frac{1}{\tilde{E}_{j+1}} \tilde{\phi}_{j+1}-\frac{\tilde{F}_{j+1}}{\tilde{E}_{j+1}} \tilde{\phi}_{j}+\frac{1}{\tilde{E}_{j}} \tilde{\phi}_{j-1}\right\rangle \\
& +\tilde{F}_{i}\left\langle\tilde{\phi}_{i-1}, \tilde{\phi}_{j}\right\rangle-\frac{\tilde{E}_{1}}{\tilde{E}_{i-1}}\left\langle\tilde{\phi}_{i-2}, \tilde{\phi}_{j}\right\rangle
\end{aligned}
$$

which finally gives us

$$
m_{i j}=\frac{\tilde{E}_{i}}{\tilde{E}_{j+1}} m_{i-1, j+1}+\frac{\tilde{E}_{i}}{\tilde{E}_{j}} m_{i-1, j-1}+\left(\tilde{F}_{i}-\frac{\tilde{E}_{i} \tilde{F}_{j+1}}{\tilde{E}_{j+1}}\right) m_{i-1, j}-\frac{\tilde{E}_{i}}{\tilde{E}_{i-1}} m_{i-2, j}
$$

This formula has to be modified for $i, j<3$, but again we omit the details. Since $m_{0 j}=\nu_{j}$ this gives us a recursive scheme to construct the matrix $M$. Again note that the derivation would become more complicated if all poles were not equal to each other.

The main reason why modified moments are used in the polynomial case is that the problem is no longer ill-conditioned. Experiments have indicated that this remains so for the rational case, with no significant loss of accuracy even for high degrees. However, we do not go into an analysis of the condition, especially since the algorithm which we present in the next section is more general and easier to analyse. Of course, as in the polynomial case this method only works well if the modified moments are available or can easily be computed. In general, it may be very difficult to obtain these modified moments.

\section{Interpolatory quadrature rules}

The name 'interpolatory quadrature formulas' usually refers to quadrature formulas where the weights are obtained by integrating some kind of interpolating function in the nodes (in our case an interpolating rational function). It therefore also covers the gaussian quadrature formulas we discussed before. However, in this section we will only be concerned with formulas which have nodes at the zeros of $\phi_{n}(x)$, but which integrate exactly with respect to a different measure $\tilde{\mu}$.

So as before let $\phi_{n}(x)$ be orthonormal with respect to $\mu$ and denote its zeros by $\left\{x_{n k}\right\}_{k=1}^{n}$. Then assume we are given a measure $\tilde{\mu}$ and define the weights

$$
A_{n k}=\int L_{n k}(x) d \tilde{\mu}(x), \quad k=1, \ldots, n
$$

where $L_{n k}(x)$ is the interpolating rational function from lemma 2.3. It is not difficult to see that the formula

$$
\sum_{k=1}^{n} A_{n k} f\left(x_{n k}\right) \approx \int f(x) d \tilde{\mu}(x)
$$

is exact for every $f \in \mathcal{L}_{n-1}$. Now we would like to know in what way these weights are related to the gaussian weights $\lambda_{n k}$. We introduce the function $\mathrm{k}_{n}(x)$ defined as

$$
\mathrm{k}_{n}(x)=\int k_{n}(x, t) d \tilde{\mu}(t) .
$$


Note that this is a function in $\mathcal{L}_{n}$. We can now prove the following simple lemma.

Lemma 5.1 Let $\mathrm{k}_{n}(x)$ be as defined above. Then we have

$$
\int \mathrm{k}_{n}(x) f(x) d \mu(x)=\int f(x) d \tilde{\mu}(x)
$$

for every $f \in \mathcal{L}_{n}$.

Proof. By the definition of $\mathrm{k}_{n}(x)$ we have

$$
\int \mathrm{k}_{n}(x) f(x) d \mu(x)=\int\left[\int k_{n}(x, t) d \tilde{\mu}(t)\right] f(x) d \mu(x) .
$$

Because of Fubini's theorem, this may be written

$$
\int \mathrm{k}_{n}(x) f(x) d \mu(x)=\int\left[\int k_{n}(x, t) f(x) d \mu(x)\right] d \tilde{\mu}(t) .
$$

The lemma now follows from the fact that $f \in \mathcal{L}_{n}$ and $k_{n}(x, t)$ is the reproducing kernel for $\mathcal{L}_{n}$.

With the aid of this lemma, it is not difficult to find the relation between $A_{n k}$ and $\lambda_{n k}$, as given in the next theorem.

Theorem 5.2 With the definitions of this section we have

$$
A_{n k}=\mathrm{k}_{n-1}\left(x_{n k}\right) \lambda_{n k}
$$

for $k=1, \ldots, n$.

Proof. The weights $A_{n k}$ were defined as

$$
A_{n k}=\int L_{n k}(x) d \tilde{\mu}(x)
$$

which by the previous lemma may be written as

$$
A_{n k}=\int \mathrm{k}_{n-1}(x) L_{n k}(x) d \mu(x) .
$$

Applying lemma 2.6 then gives

$$
A_{n k}=\mathrm{k}_{n-1}\left(x_{n k}\right) \int L_{n k}(x) d \mu(x)
$$

which, according to equation (4), proves the theorem.

The previous theorem shows that the interpolatory weights can be computed from the gaussian weights as soon as we have obtained the function $\mathrm{k}_{n}(x)$.

The relation to modified moments follows from the observation that

$$
\mathrm{k}_{n}(x)=\sum_{k=0}^{n} \tilde{\nu}_{k} \phi_{k}(x)
$$

where

$$
\tilde{\nu}_{k}=\int \phi_{k}(x) d \tilde{\mu}(x) .
$$


Of course, switching the role of $\mu$ and $\tilde{\mu}$ gives us a quadrature formula based on the modified moments $\nu_{k}$ defined in the previous section, which integrates with respect to $\mu$. Since the inner products in lemma 2.2 are integrals with respect to $\mu$, we can use this interpolatory quadrature formula to compute the recurrence coefficients $A_{k}$ and $B_{k}$ (or equivalently $E_{k}$ and $F_{k}$ ). However, the integrands are functions in $\mathcal{L}_{n} \cdot \mathcal{L}_{n-1}$, which means that we need $2 n$ modified moments $\left\{\nu_{k}\right\}_{k=0}^{2 n-1}$ and the gaussian quadrature formula associated to $\tilde{\phi}_{2 n}$ and the pole sequence $\left\{\alpha_{1}, \alpha_{1}, \alpha_{2}, \alpha_{2}, \ldots\right\}$, to compute $\left\{A_{k}, B_{k}\right\}_{k=0}^{n-1}$. So we have to double the multplicity of every pole. If we assume that the gaussian quadrature formula and the modified moments are obtained with machine precision, then the only loss of accuracy is in the evaluation of the sum (12) and in applying the quadrature formula to the inner products. Since both the modified moments and the normalized orthogonal rational functions usually remain small, hardly any digits are lost this way. The following example gives an illustration.

Example 5.3 Take poles at the integer multiples of $\omega=1.001$, so we have

$$
A=\{\omega,-\omega, 2 \omega,-2 \omega, \ldots\} .
$$

The functions we wish to compute are orthogonal with respect to the normalized Lebesgue measure on $[-1,1]$,

$$
d \mu(x)=\frac{1}{2} d x
$$

and we will use Chebyshev orthogonal rational functions $\tilde{\phi}_{n}$ to compute them, which means that

$$
d \tilde{\mu}(x)=\frac{1}{\pi} \frac{d x}{\sqrt{1-x^{2}}} .
$$

For more details about these functions we refer to [28]. We can compute the modified moments using the formulas from that article combined with those from [34]. If we take $n=20$ then we need the gaussian nodes and weights associated to $\tilde{\phi}_{40}$ to compute $\left\{A_{k}, B_{k}\right\}_{k=0}^{19}$. Figure 1 shows the error growth for the coefficient $B_{k}$ using modified moments (solid line) and ordinary moments (dashed line) as a function of $k$. Note that we can indeed compute the coefficients up to machine precision using the algorithm based on modified moments. Only when $k \geq n$ the error increases rapidly (which is not surprising, since the quadrature formula is no longer exact now; if we want more coefficients, we need more modified moments).

As we mentioned before, the nodes and weights for the gaussian quadrature can be computed from the recurrence coefficients by solving a generalized eigenvalue problem. This means that, given the modified moments $\nu_{k}$ and the coefficients $\left\{\tilde{A}_{k}, \tilde{B}_{k}\right\}$, we can easily compute the interpolatory quadrature rule and thus the coefficients $\left\{A_{k}, B_{k}\right\}$.

\section{Conclusion}

We presented some moment-based algorithms for computing orthogonal rational functions. In the case of ordinary (rational) moments, a detailed error analysis showed that this is an ill-conditioned problem, just like in the polynomial case. The polynomial algorithm based on modified moments can only be generalized to the rational case if we assume that all poles are equal, which is a severe restriction. To overcome this problem, we developed a new algorithm using modified moments, based on interpolatory quadrature formulas. This algorithm works for arbitrary poles. To our knowledge, the connection between modified moments and interpolatory quadrature has not been exploited before in this way, the reason probably being that in the polynomial case, there is no need for a second algorithm for modified moments, since all poles are equal to each other (at infinity). 


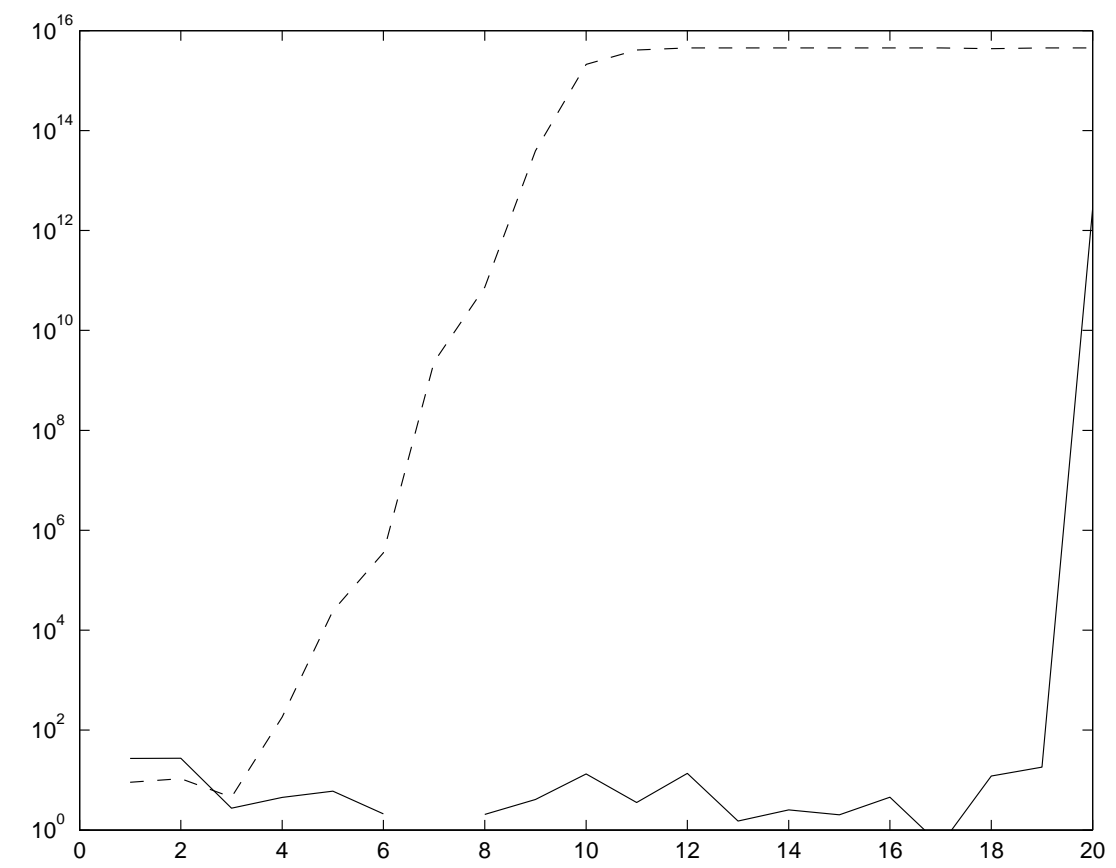

Fig. 1 Error growth in $B_{k}$ using modified (solid) and ordinary (dashed line) moments.

\section{References}

[1] A. Bultheel and P. González Vera. Wavelets by orthogonal rational kernels. In B. Berndt and F. Gesztesy, editors, Continued fractions: from number theory to constructive approximation, volume 236 of Contemporary Mathematics, pages 101-126. AMS, 1999.

[2] A. Bultheel and P. González Vera. Rational wavelets on the real line. Numer. Funct. Anal. Optim., 21(1-2):77-96, 2000.

[3] A. Bultheel, P. González Vera, E. Hendriksen, and O. Njåstad. Orthogonal Rational Functions, volume 5 of Cambridge Monographs on Applied and Computational Mathematics. Cambridge University Press, 1999.

[4] A. Bultheel, P. González Vera, E. Hendriksen, and O. Njåstad. Orthogonal rational functions and continued fractions. In S. Suslov, editor, Special functions 2000, NATO-ASI, Tempe, 2000, Communications in the Analytic Theory of Continued Fractions. Kluwer, 2000.

[5] A. Bultheel, P. González Vera, E. Hendriksen, and O. Njåstad. Orthogonal rational functions and quadrature on the real half line. J. Complexity, 19(3):212-230, 2002.

[6] A. Bultheel, P. González Vera, E. Hendriksen, and O. Njåstad. Orthogonal rational functions and tridiagonal matrices. J. Comput. Appl. Math., 153(1-2):89-97, 2003.

[7] A. Bultheel, P. González Vera, E. Hendriksen, and O. Njåstad. Orthogonal rational functions on the real half line with poles in $[-\infty, 0]$. J. Comput. Appl. Math., 2004. Accepted.

[8] A. Bultheel and P. Van gucht. Boundary asymptotics for orthogonal rational functions on the unit circle. Acta Applicandae Mathematicae, 61:333-349, 2000.

[9] W. Gautschi. Construction of Gauss-Christoffel quadrature formulas. Math. Comp., 22:251-270, 1968.

[10] W. Gautschi. On the Construction of Gaussian Quadrature Rules from Modified Moments. Math. Comp., 24:245$260,1970$.

[11] W. Gautschi. On generating orthogonal polynomials. SIAM J. Sci. Stat. Comput., 3:289-317, 1982.

[12] W. Gautschi. Orthogonal polynomials: applications and computation. Acta Numerica, 5:45-119, 1996.

[13] X. Li. Regularity of orthogonal rational functions with poles on the unit circle. J. Comput. Appl. Math., 105:371383, 1999.

[14] X. Li and K. Pan. Strong and weak convergence of rational functions orthogonal on the unit circle. J. London Math. Soc., 53:289-301, 1996. 
[15] I.P. Mysovskih. On the construction of cubature formulas with fewest nodes. Sov. Math., Dokl., 9:277-280, 1968.

[16] B. Ninness. Frequency domain estimation using orthonormal bases. In Proceedings of the 13th IFAC World Congress, volume 8, pages 381-386, San Francisco, 1996.

[17] B. Ninness and H. Akçay. Rational basis functions for robust identification from frequency and time domain measurements. Automatica, vol. 34, no. 9:1101-1117, 1998.

[18] B. Ninness and F. Gustafsson. A unified construction of orthogonal bases for system identification. IEEE Trans. Automat. Control, 42:515-522, 1997.

[19] K. Pan. Extensions of Szegő's theory of rational functions orthogonal on the unit circle. J. Comput. Appl. Math., 62:321-331, 1995.

[20] K. Pan. Convergence of orthogonal rational functions outside of the unit circle. J. Comput. Appl. Math., 69:345351, 1996.

[21] K. Pan. On the convergence of rational functions orthogonal on the unit circle. J. Comput. Appl. Math., 76:315324, 1996.

[22] K. Pan. The zero distribution of orthogonal rational functions on the unit circle. Can. J. Math., 49:810-819, 1997.

[23] J. Van Deun and A. Bultheel. Orthogonal rational functions and quadrature on an interval. J. Comput. Appl. Math., 153(1-2):487-495, 2003.

[24] J. Van Deun and A. Bultheel. Ratio asymptotics for orthogonal rational functions on an interval. J. Approx. Theory, 123:162-172, 2003.

[25] J. Van Deun and A. Bultheel. The computation of orthogonal rational functions on an interval. J. Comput. Appl. Math., 2004. Accepted.

[26] J. Van Deun and A. Bultheel. An interpolation algorithm for orthogonal rational functions. J. Comput. Appl. Math., 164-165:749-762, 2004.

[27] J. Van Deun and A. Bultheel. A weak-star convergence result for orthogonal rational functions. J. Comput. Appl. Math., 2004. Accepted.

[28] J. Van Deun, A. Bultheel, and P. González Vera. On computing rational Gauss-Chebyshev quadrature formulas. Math. Comp., 2004. Accepted.

[29] P. Van gucht and A. Bultheel. A relation between orthogonal rational functions on the unit circle and the interval $[-1,1]$. Comm. Anal. Th. Continued Fractions, 8:170-182, 2000.

[30] P. Van gucht and A. Bultheel. State space representation for arbitrary orthogonal rational functions. Systems Control Lett., 49(2):91-98, 2003.

[31] P. Van gucht and A. Bultheel. Using orthogonal rational functions for system identification. IEEE Trans. Automat. Control, 48(4):705-709, 2003.

[32] B. Wahlberg. System identification using Laguerre models. IEEE Trans. Automat. Control, vol. AC-36, no. 5:551-562, 1991 .

[33] B. Wahlberg. System identification using Kautz models. IEEE Trans. Automat. Control, vol. AC-39, no. 6:12761282, 1994.

[34] J.A.C. Weideman and D.P. Laurie. Quadrature rules based on partial fraction expansions. Numer. Algorithms, 24:159-178, 2000. 